\title{
SETS OF POINTS OF DISCONTINUITY
}

\author{
RICHARD BOLSTEIN
}

ABstract. In order that a subset $F$ of a topological space coincide with the set of points of discontinuity of a real-valued function on the space, it is necessary that $F$ be an $F_{\sigma}$-set devoid of isolated points. It is shown that this condition is also sufficient if the space is "almost-resolvable", and in particular if the space is either separable, first countable, locally compact Hausdorff, or topological linear.

1. Introduction. It is well known that the set of points of discontinuity of a real-valued function on a topological space $X$ belongs to the class $F_{\sigma}$ of countable unions of closed sets. An outline of the proof can be found in Hewitt and Stromberg [3, p. 78]. It is obvious that such a set can contain no isolated points of $X$. It is natural to ask this question: Does every $F_{\sigma}$-subset which contains no isolated points of $X$ coincide with the set of points of discontinuity of some real-valued function on $X$ ?

An affirmative answer to this question was given in the case of the realline by W. H. Young [4] in 1907. In 1932, H. Hahn [1, p. 193] showed that in fact any metric space has this property. In this article we give an affirmative answer to the question for a large class of topological spaces, which includes, in particular, any space which is either first countable, separable, locally compact Hausdorff, or topological linear. Moreover, we characterize those $F_{\sigma}$-subsets of an arbitrary space which coincide with the set of points of discontinuity of a function with countable range.

In the next section we introduce the concept of "almost-resolvable" spaces, which is a generalization of the so-called resolvable spaces of E. Hewitt. The main results appear in $\S 3$.

2. Almost-resolvable spaces. Hewitt [2] calls a topological space resolvable if it is the union of two disjoint dense sets. In [2, p. 331] he shows that

(a) a first countable space devoid of isolated points is resolvable, and

(b) a locally compact Hausdorff space devoid of isolated points is resolvable.

Received by the editors April 10, 1972.

AMS (MOS) subject classifications (1970). Primary 54-XX, 54C30, 26A15; Secondary $54 \mathrm{C} 50$

Key words and phrases. $D F_{\sigma}$-space, resolvable, almost-resolvable, $S I$-space.

(c) American Mathematical Society 1973 
We can now show that

(c) a linear topological space over a nondiscrete valuated field is resolvable.

For let $K$ be a valuated field, that is, a field which admits an absolute value function $a \rightarrow|a|$ of $K$ into the nonnegative reals such that $|a+b| \leqq$ $|a|+|b|$ and $|a b|=|a||b|$ for all $a, b$ in $K$, and such that $|a|=0$ if, and only if, $a=0$. Then $(a, b) \rightarrow|a-b|$ defines a metric on $K$, and if this metric is nondiscrete, then $K$ has no isolated points in the metric topology. So by (a) above, $K$ is resolvable. Let $K_{1}$ and $K_{2}$ be disjoint dense sets in $K$. Let $X$ be a linear topological space over $K$, and $B$ be any Hamel basis for $X$. It follows from the continuity of addition and scalar multiplication that the sets $D_{1}$ and $D_{2}$ of finite linear combinations of elements of $B$ with coefficients in $K_{1}$ and $K_{2}$, respectively, are disjoint and dense in $X$.

In Theorem 4 below we will require a slight generalization of resolvability. We first give a new characterization of resolvability.

THEOREM 1. A topological space is resolvable if, and only if, it is a finite union of sets with void interiors.

Proof. The necessity is trivial. Assume now that $X=D_{1} \cup \cdots \cup D_{n+1}$ where each $D_{k}$ has void interior. On replacing each $D_{j}$ by $D_{j} \mid \cup_{k<j} D_{k}$ we can assume that the sets are pairwise disjoint. If $n=1$, then $D_{1}$ and $D_{2}$, each having void interior, are dense, so $X$ is resolvable. Assume now that any space which is the union of $n$ sets with void interiors is resolvable. Let $U \subset X$ be any nonvoid open set. If $D_{n+1}$ is dense in $U$, then $U$ is resolvable (in the relative topology) since $D_{n+1} \cap U$ has void interior in $U$. Otherwise, there is a nonvoid open set $V \subset U$ disjoint from $D_{n+1}$. Since $V=\left(D_{1} \cap V\right) \cup$ $\cdots \cup\left(D_{n} \cap V\right)$, it is resolvable by the induction hypothesis. We have shown that every nonvoid open set contains a nonvoid open resolvable subset. By [2, Theorem 20], $X$ is resolvable.

DEFINITION. A topological space will be called almost-resolvable if it is a countable union of sets with void interiors.

Clearly, a resolvable space is almost-resolvable, and an almostresolvable space has no isolated points. Note that if a space $X$ contains a dense set which is a countable union of sets with void interiors, then $X$ is almost-resolvable. It follows that

(d) a separable space with no isolated points is almost-resolvable.

We now construct examples of almost-resolvable spaces which are not resolvable. Following Hewitt [2], we call a topological space an SIspace if it has no isolated points and if no nonempty subset is resolvable in the relative topology.

THEOREM 2. (A) There exists a separable $T_{1}$-space of any prescribed infinite cardinality which has no isolated points but is not resolvable. (It is 
almost-resolvable by (d).) (B) There exists a completely regular Hausdorff space of any prescribed infinite cardinality which is an SI-space but is almost-resolvable.

Proof. First recall that if $\left(X_{\alpha}\right)$ is any family of pairwise disjoint topological spaces, the free union of this family is the space $X=\bigcup X_{\alpha}$ where a set $U \subset X$ is open if, and only if, $U \cap X_{\alpha}$ is open in $X_{\alpha}$ for every $\alpha$. Since each $X_{\alpha}$ is open in $X$, a set $D \subset X$ is dense in $X$ if, and only if, $D \cap X_{\alpha}$ is dense in $X_{\alpha}$ for every $\alpha$. It follows that $X$ is resolvable (almostresolvable) if, and only if, each $X_{\alpha}$ is resolvable (almost-resolvable). Also, $X$ is an $S I$-space if, and only if, each $X_{\alpha}$ is an $S I$-space.

(A) Let $X_{1}$ be any infinite set, and let $D \subset X$ be a countably infinite subset. Define a topology on $X_{1}$ by declaring a set to be open if, and only if, it contains all but finitely many elements of $D$. It is clear that, in this topology, $X_{1}$ is a $T_{1}$-space devoid of isolated points, and since $D$ is dense by construction, $X_{1}$ is separable.

Now let $X_{2}$ be any countably infinite set disjoint from $X_{1}$. By [2, Theorem 25], $X_{2}$ can be endowed with a topology in which it is $T_{2}$ and $S I$. Let $X$ be the free union of $X_{1}$ and $X_{2}$. Then $\operatorname{card}(X)=\operatorname{card}\left(X_{1}\right) . X$ is separable, $T_{1}$, and devoid of isolated points, because $X_{1}$ and $X_{2}$ have these properties. Since $X_{2}$ is not resolvable, $X$ is not resolvable.

(B) Let $J$ be any infinite set. For each $\alpha \in J$, let $Y_{\alpha}$ be a countably infinite set and let $X_{\alpha}=\{\alpha\} \times Y_{\alpha}$. Then the sets $X_{\alpha}$ are countably infinite and pairwise disjoint. By [2, Theorem 27], each $X_{\alpha}$ can be endowed with a completely regular, Hausdorff, $S I$ topology. Let $X$ be the free union of the family $\left(X_{\alpha}\right)_{\alpha \in J}$. Then $\operatorname{card}(X)=\operatorname{card}(J)$. It is easy to verify that $X$ is a completely regular, Hausdorff space. Since each $X_{\alpha}$ is an $S I$-space, $X$ is an $S I$-space. Since each $X_{\alpha}$, as a countable space with no isolated points, is trivially almost-resolvable, $X$ is almost-resolvable.

REMARK. In part (A) of Theorem 2, we can replace $T_{1}$ by Hausdorff if the prescribed cardinality is aleph null, $c$, or $2^{c}$. (It is well known that a separable Hausdorff space has cardinality at most $2^{c}$.) For in the proof of (A), one can take for $X_{1}$, the rationals, $[0,1]$, or $[0,1]^{[0,1]}$ with the usual topologies.

3. $D F_{\sigma}$-spaces. We first characterize those $F_{\sigma}$-subsets of an arbitrary space which coincide with the set of points of discontinuity of a function with countable range.

THEOREM 3. Let $F$ be an $F_{\sigma}$-subset of a topological space $X$. In order that $F$ coincide with the set of points of discontinuity of a real-valued function $g$ on $X$ such that $g(F)$ is countable, it is necessary and sufficient that $F$ be a countable union of sets with void interiors. In this case, the function $g$ can be chosen so that $g(X)$ is countable. 
Proof. For the necessity, assume that there exists a function $g$ on $X$ whose set of discontinuities is precisely $F$, and such that $g(F)$ is countable. Then if $r \in g(F)$, the set $F \cap g^{-1}(r)$ has void interior (otherwise $g$ would have a point of continuity in $F$ ). Therefore

$$
F=\bigcup\left\{F \cap g^{-1}(r): r \in g(F)\right\}
$$

is a countable union of sets with void interiors.

For the sufficiency, first note that any $F_{\sigma}$-set $F$ can be written in the form $F=\bigcup E_{n}$, where $\left\{E_{n}: n=1,2, \cdots\right\}$ is a countably infinite collection of pairwise disjoint sets such that $F_{n}=E_{1} \cup \cdots \cup E_{n}$ is closed for every $n$. Since we assume that $F$ is a countable union of sets with void interiors, the same is true for every subset of $F$. In particular, for each $n$ there is a countable collection $\left\{E_{m n}: m=1,2, \cdots\right\}$ of pairwise disjoint sets with void interiors such that $E_{n}^{\circ}=\bigcup_{m} E_{m n}$. The required function $g$ is defined by

$$
\begin{aligned}
g(x) & =0: \quad x \notin F, \\
& =1 / n: \quad x \in E_{n} \backslash E_{n}^{\circ} ; \quad n=1,2, \cdots, \\
& =1 /(n+m): \quad x \in E_{m n} ; \quad m, n=1,2, \cdots .
\end{aligned}
$$

Now $g$ is clearly continuous on $X \backslash F$, for if $x \notin F$ then, for each $n, X \backslash F_{n}$ is a neighborhood of $x$ on which $|g|<1 / n$.

Since $g(F) \subset\{1 / n: n=1,2, \cdots\}$ is discrete, to show that $g$ is discontinuous at every point of $F$ it suffices to show that $g$ is not constant on any open set $V$ which meets $F$. Assume then that $V$ is open and $V \cap E_{n} \neq \varnothing$ for some $n$.

If $V \cap E_{n}^{\circ} \neq \varnothing$, then since each $E_{m n}$ has void interior, $V$ meets $E_{m n}$ for at least two values of $m$, and hence $g$ is not constant on $V$ in this case. If $V \cap E_{n}^{\circ}=\varnothing$, then $V$ contains a point $x \in E_{n} \backslash E_{n}^{\circ}=E_{n} \cap \operatorname{bd}\left(E_{n}\right)$. Since $V \cap\left(X \backslash F_{n-1}\right)$ is a neighborhood of $x$, it must meet $X \backslash E_{n}$, and hence $V$ meets $\left(X \backslash F_{n-1}\right) \cap\left(X \backslash E_{n}\right)=X \backslash\left(F_{n-1} \cup E_{n}\right)=X \backslash F_{n}$. Since $g<1 / n$ on $X \backslash F_{n}$ and $g=1 / n$ on $E_{n} \backslash E_{n}^{\circ}, g$ is not constant on $V$ in this case either, so the proof is complete.

COROLlary 1. In an arbitrary topological space $X$, any $F_{\sigma}$-set of the first category in $X$ coincides with the set of points of discontinuity of some real-valued function on $X$.

COROllary 2. A topological space $X$ is almost-resolvable if, and only if, there is an everywhere discontinuous function on $X$ with countable range.

Definition. A topological space $X$ will be called a $D F_{\sigma}$-space if every $F_{\sigma}$-subset devoid of isolated points of $X$ coincides with the set of points of discontinuity of some real-valued function on $X$. 
Let $S$ denote the set of isolated points of the space $X$, and let $Y=X \backslash \bar{S}$. Then $Y$ has no isolated points.

THEOREM 4. If $Y$ is almost-resolvable, then $X$ is a $D F_{\sigma}$-space.

Proof. Let $F$ be an $F_{\sigma}$-subset of $X$ disjoint from $S$. Then $F^{\circ} \cap S=\varnothing$, so $\bar{S} \subset X \backslash F^{\circ}$ and $F^{\circ} \subset Y$. Since an open subset of an almost-resolvable space is almost-resolvable, $F=F^{\circ} \cup\left(F \backslash F^{\circ}\right)$ is a countable union of sets with void interiors, so the result follows from Theorem 3.

COROLlaRY 3. If $Y$ is of the first category in itself, then $X$ is a DF-space.

Corollary 4. The class of $D F_{\sigma}$-spaces contains

(a) any first-countable space,

(b) any locally compact Hausdorff space,

(c) any separable space,

(d) any linear topological space.

Proof. This follows from Theorem 4 and (a)-(d) of $\S 2$, since the set $Y$ is open and therefore inherits properties (a), (b), or (c) from $X$.

The author wishes to thank B. J. Pettis for several helpful conversations.

\section{REFERENCES}

1. H. Hahn, Reelle Funktionen, Akademie Verlagsgesellschaft, Leipzig, 1932; reprint, Chelsea, New York, 1948.

2. E. Hewitt, A problem of set-theoretic topology, Duke Math. J. 10 (1943), 309-333. MR 5, 46.

3. E. Hewitt and K. Stromberg, Real and abstract analysis. A modern treatment of the theory of functions of a real variable, Springer-Verlag, New York, 1965. MR 32 \#5826.

4. W. H. Young, Über die Einteilung der unstetigen Funtionen und die Verteilung ihrer Stetigkeitspunkte, S.-B. Akad. Wiss. Wien Math.-Natur. K. Abt. IIA 112 (1907), 13071311.

Department of Mathematics, University of North Carolina at Chapel Hill, Chapel Hill, North Carolina 27514 